\title{
Research on Urban Traffic Active Control in Cooperative Vehicle Infrastructure
}

\author{
Li-li Zhang $\mathbb{D}^{1},{ }^{1,2}$ Li Wang, ${ }^{2}$ Qi Zhao $\mathbb{D}^{\circ},{ }^{2}$ Fang Wang, ${ }^{1}$ Yadongyang Zhu, ${ }^{1}$ and Ling-yu Zhang ${ }^{2}$ \\ ${ }^{1}$ College of Information Engineering, Beijing Institute of Petrochemical Technology, Beijing 102617, China \\ ${ }^{2}$ Beijing Key Lab of Urban Intelligent Control Technology, North China University of Technology, Beijing 100144, China
}

Correspondence should be addressed to Li-li Zhang; zllphd2012@163.com

Received 26 March 2021; Revised 6 April 2021; Accepted 13 April 2021; Published 21 April 2021

Academic Editor: Elżbieta Macioszek

Copyright (c) $2021 \mathrm{Li}$-li Zhang et al. This is an open access article distributed under the Creative Commons Attribution License, which permits unrestricted use, distribution, and reproduction in any medium, provided the original work is properly cited.

Urban intersection control mainly undertakes two tasks: traffic safety and traffic efficiency. Traditional intersection control models and methods have been insufficient in improving traffic efficiency, which is composed of the increase in traffic demand and the complexity of demand at present. In this paper, we propose a novel model and method called ATCM, which is based on the advanced technology of cooperative vehicle infrastructure. In this paper, a novel active traffic control model (ATCM) is proposed, which is based on the advanced technology of cooperative vehicle infrastructure. ATCM increases the intersection control model variables from the traditional two dimensions to five dimensions. It reshapes intersection control from the perspective of road designers and managers, so it can achieve more flexible and efficient traffic control. To this end, a multivariable active traffic control model is constructed, which includes road speed, lane control, sequence, phase, and green light time; a D-double layer optimization method is designed for this model. The first part of this optimization method combines speed control and dynamic phase sequence control. The second part is realized by the combination of lane control and dynamic phase sequence control. By conducting comprehensive experiments, the results demonstrate that the proposed approach is more flexible and efficient than traditional methods.

\section{Introduction}

The essence of traffic flow change is that the traffic demand matches the space-time resources. The traffic control at the intersection is a way to allocate space-time resources based on ensuring traffic safety. Therefore, the space-time resource variables which can accurately describe the characteristics of intersections directly affect the flexibility and advancement of traffic control.

Today, the most widely used traffic signal control systems (such as SCOOT $[1,2]$ and SCATS $[3,4]$ ) use the expert experience to design static variables (lanes, phases, and phase sequences) at intersections and then use models and algorithms to implement the control strategy with the green light time as the main variable. However, the dynamic characteristics of the traffic flow often do not match well with the capacity of the road, which makes the traditional traffic control based on the allocation of time resources difficult to effectively allocate road space resources. Unfortunately, many road congestions are caused by insufficient space resource allocation.

In recent years, with the development of technologies such as cooperative vehicle infrastructure $[5,6]$ and autonomous driving [7-9], it has become possible to allocate space resources in real time; and what is encouraging is that it can reshape the traffic flow by changing the distribution of traffic demand when spatial resources really cannot meet the traffic demand of overload. This happens to solve a significant problem: "The shape of urban intersections, the number of lanes, and lane flow are designed based on historical traffic data and survey data. The designer of the intersection control strategy carries out signal control on its basis. However, it is worth thinking about that the growth of cars in many cities is very fast, and the design limit capacity of intersections will soon be broken. Some intersections have problems at the beginning of the operation. These problems 
have caused the design of intersections to fail to meet the needs of increasing traffic flow, and also lead to the failure of traffic control."

Surprisingly, although the new technology has been widely used in the field of traffic control $[10,11]$, it is still mainly based on the allocation of time resources in the traditional theoretical framework of traffic control, and no one has tried to increase the dimension and further research on extended control capabilities. In this paper, a multivariable active control model for intersections is proposed, which is fully considered in the cooperative vehicle infrastructure technology. More specifically, a multivariable control model and optimization method including vehicle speed, lane, phase, phase sequence, and green light time is designed. It can reshape the traffic flow through control. It is called active control to distinguish the passive control of traditional traffic. The definition of active control is as follows: "Active control is the control that can influence the whole process of traffic flow from forming to passing through the intersection in terms of time and space." Then traditional traffic control models and methods can be called passive control because some control methods can only affect the passage of traffic flow in time or space, while others can only affect the formation of traffic flow.

The main contributions of this paper include the following:

(i) The multivariable active control model is a new intersection control model. It can not only adapt to traffic demand but also actively change traffic demand.

(ii) This model can effectively adapt and change different traffic flow requirements through the control of vehicle speed variables and lane variables to reduce the problem of intersection congestion caused by defects in intersection design.

(iii) This model allows designers and implementers of control algorithms to have greater flexibility and can create more optimized algorithms in cooperative vehicle infrastructure.

The remainder of this paper is organized as follows: Section 2 presents a literature review of related work. Section 3 proposes a multivariable active control model for intersections. Section 4 designs a D-double layer optimization method for the active control model. The first part of this optimization method combines speed control and dynamic phase sequence control. The second part is realized by the combination of lane control and dynamic phase sequence control. Section 5 describes the simulation experiments and presents the experimental results. Section 6 concludes this paper and discusses directions for future research.

\section{Literature Review}

Urban intersections control is an age-old issue that goes back to the 1950s [12]. This is the most important issue in the field of urban traffic, even in the future of vehicle-road collaboration and the popularity of autonomous driving.
Therefore, it has attracted the attention of many scholars. Existing methods can be divided into two categories.

2.1. Passive Traffic Control. Based on the traditional urban road traffic control theory, combining models and algorithms of modern control, intelligent control, and other theories have achieved considerable development and application in the field of traffic control. Modern control theory assumes that the mathematical model of the controlled object is known. Traffic control methods based on modern control theory are mostly called model-based traffic control theory and methods $[10,11,13-16]$. At the beginning of this century, the advancement of traffic information and the development of detection technology have greatly improved the types and accuracy of traffic detection data. At the same time, the explosive growth of road traffic travel requirements has made traditional traffic control methods stretched. People began to think about the data-driven traffic control theory and method [17], because when it is difficult to develop a model for a controlled system, the system input and output data can be used to implement control and decision-making.

2.2. Active Traffic Control. The abundance of traffic control data benefited from the development of information technology, and the theory of traffic control may therefore be changed. Therefore, some studies have proposed active traffic control. At present, there are two main types of research on active traffic control at intersections: One is the active control based on traffic flow prediction, which is realized by building a traffic flow prediction model combined with traditional traffic control [18-21]. The other is active control based on cooperative vehicle infrastructure, which combines vehicle speed guidance and traffic control to achieve active control through the use of cooperative vehicle infrastructure information [22-25].

However, to the best of the authors' knowledge, it is difficult to obtain satisfactory results in terms of the effectiveness and flexibility of current and future intersection control. There are two main reasons: One is that the limit capacity considered in the design of the intersection will soon be broken, and some intersections have problems at the beginning of the design. The existing research traffic control models and methods hardly consider this problem, so they often cause traffic control failures in practical applications. The other is that both traditional traffic control and active control are based on traditional traffic control theories. They only use phase, sequence, and green light time as control variables and their scalability and flexibility are not high. As a result, the application of traffic control in the development of technologies such as cooperative vehicle infrastructure and autonomous driving is restricted. It can be said that the collision between the flexibility of new theories and new technologies and the limitations of traditional traffic control cannot give full play to its true capabilities in the fettered traffic control system. Therefore, an effective way to solve this problem is to expand the set of control variables and to effectively allocate road time and space resources as the goal 
to build a multivariable active traffic control model at intersections.

\section{Active Control Model of Intersection}

The intersection active traffic control model is composed of a time-space resource scheduling model and speed control model. The model is divided into two parts, the speed control area and the lane control area, as shown in Figure 1.

The length of the lane control area is $L_{j, a}$, and the length of the vehicle speed control area is $L_{j, b}$, as shown in Figure 2.

3.1. Time-Space Resource Scheduling Model. The intersection consists of an internal conflict area and upstream and downstream connecting sections, as shown in Figure 3. Consider the state formula of link $j$ in the set of connected links at the intersection by formula (1).

$$
n_{j, a}(k+1)=n_{j, a}(k)+q_{j, a, \text { in }}(k)-q_{j, a, \text { out }}(k) .
$$

In the formula, $n_{j, a}(k)$ is the number of vehicles in period $k$ on road $j, a, q_{j, a, \text { in }}(k)$ is the number of vehicles in period $k$ entering road $j, a$, and $q_{j, a, \text { out }}(k)$ is the number of vehicles in period $k$ leaving road $j, a$.

3.1.1. The Space Variables [26]. To accurately characterize the dynamic characteristics of lane properties, the paper first proposes the concept of "lane genes." Then the turning properties of the lane change into a control variable. As shown in Figure 4(a), the turning properties of the lane include left turn, straight turn, and right turn, which are described as $L, T$, and $R$.

$$
\Gamma_{j, a}(k)=\left\{\varpi_{j, a, o}(k)\right\}_{o=1,2, \ldots, \omega_{j, a}(k)},
$$

Further, the smallest unit of traffic scheduling at the intersection is composed of the turning attribute of the entrance lane of the intersection and the downstream link. As shown in Figure 4(b), $j, a$ is the upstream road and $o$ is the downstream road. $F_{j, a}(k)=\left\{f_{r}^{(j, a)}(k)\right\}_{r=1,2, \ldots, m}$ is the lane gene expression combination in period $k$, where $f_{r}^{(j, a)}(k)$ represents the gene expression of lane $r$, $f_{r}^{(j, a)}(k)=\left\{G_{u}^{(j, a \longrightarrow r)}(k)\right\}_{u=1,2,3}$, where $G_{u}^{(j, a \longrightarrow r)}(k)$ is the gene of lane $r$ and $G_{u}^{(j, a \rightarrow r)}(k)=\left\{\begin{array}{ll}0, & \text { if Gene doesn't be express } \\ 1, & \text { Gene is express }\end{array}\right.$. A lane consists of three genes: $G_{1}, G_{2}$, and $G_{3} . G_{1} \longrightarrow L$ means that the first gene map is turned left, $G_{2} \longrightarrow T$ means that the second gene map goes straight, and $G_{3} \longrightarrow R$ means that the third gene map is turned right.where $\Gamma_{j, a}(k)$ is the set of spatial variables. $\Phi_{j, a, o}(k)$ is the control variable, which is a function of the number of lanes, as shown in formula (3). $\omega_{j, a}(k)=\sum_{u=1}^{3} G_{F_{j, a}(k)[\cup](u)}$, and $\omega_{j, a}(k)$ is the number of connections from road $j, a$ to $\operatorname{road} o . F_{j, a}(k)[U]=$ $f_{r=1}^{(j, a)}(k) \cup f_{r=2}^{(j, a)}(k) \cup \cdots \cup f_{r=m}^{(j, a)}(k), G_{F_{j, a}(k)[\cup](1)}$ is the first gene in the lane set gene expression, $G_{F_{j, a}(k)[U](2)}$ is the

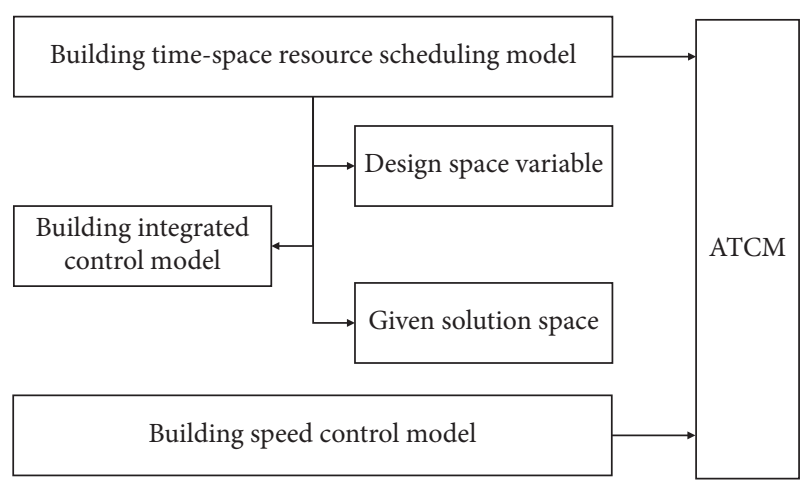

FIGURE 1: Building active traffic control model of intersection.

second gene in the lane set gene expression, and $G_{F_{j, a}(k)[U](3)}$ is the third gene in the lane set gene expression.

$$
\begin{cases}\varpi_{j, a, o}(k)=\frac{\omega_{o}^{j, a}(k)}{m}, & \text { s.t. } 0<\varpi_{j, a, o}(k) \leq 1, \\ \omega_{o}^{j, a}(k)=\left\{\sum_{i=1}^{m} G_{i, u}^{j, a \longrightarrow o}\right\}_{u=1,2,3}, & \end{cases}
$$

where $\Phi_{o}^{j, a}(k)$ is the number of identical genes after lane gene expression.

3.1.2. Integrated Model. From formulas (1) and (2), formula (4) can be obtained:

$$
\begin{aligned}
n_{j, a}(k+1)= & n_{j, a}(k)+q_{j, a, \text { in }}(k) \\
& -\sum_{o=0}^{\min \left\{\omega_{j, a}(k), \omega_{j, a}^{X x}(k)\right\}} \omega_{j, a, o}(k) \cdot S_{j, a} \cdot g_{j, a, o}(k),
\end{aligned}
$$

where $S_{j, a}$ is the capacity of the road section, $g_{j, a, o}(k)$ is the green light time of the phase of the road section $j, a$ in the sampling period $k$, and $g_{j, a, o}(k) \geq g_{j, a, \min } \cdot g_{j, a, o}(k)$ is obtained from the following solution space.

3.1.3. Solution Space. The expression set of lane genome in all directions of the entrance section of the intersection is as follows:

$$
\Phi_{x}(k)=\left\{F_{I}^{(x)}(k)\right\}_{I=1,2, \ldots, \varepsilon^{*}}
$$

A set of phase combinations of lanes in all directions of the entrance section of an intersection is as follows.

When expressed in a fixed genome,

$$
P_{\Phi_{x}}(k)=\left\{O_{I I}^{(\Phi x)}(k)\right\}_{I I=1,2, \ldots, \sigma^{\circ}} .
$$




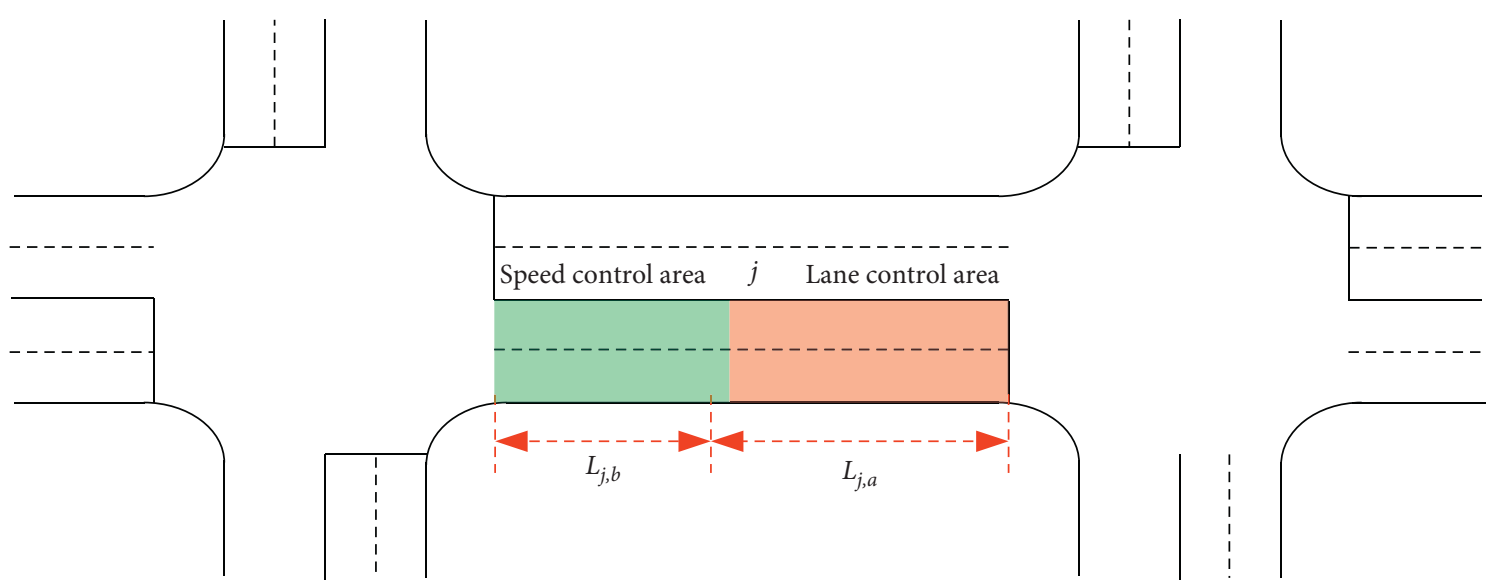

FIGURE 2: Active traffic control model of intersection.

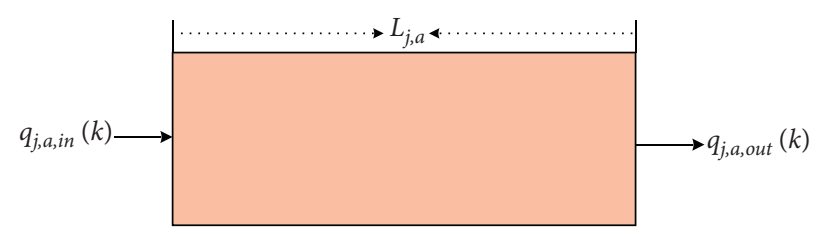

Figure 3: Storage and forwarding model of intersection.

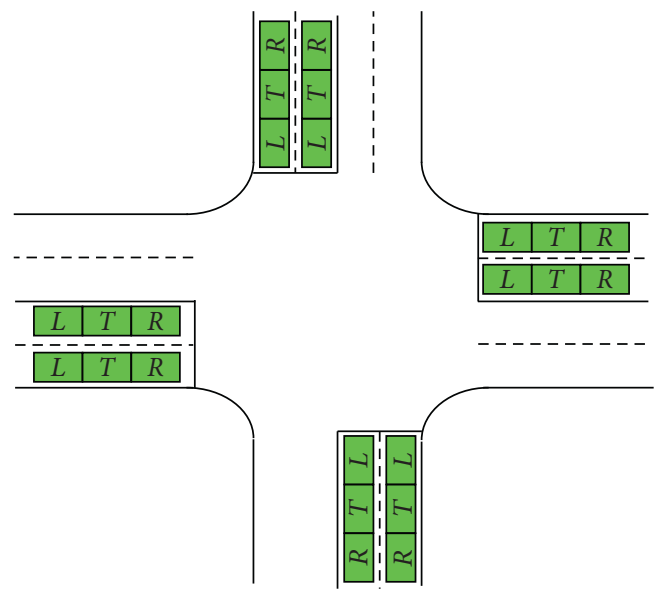

(a)

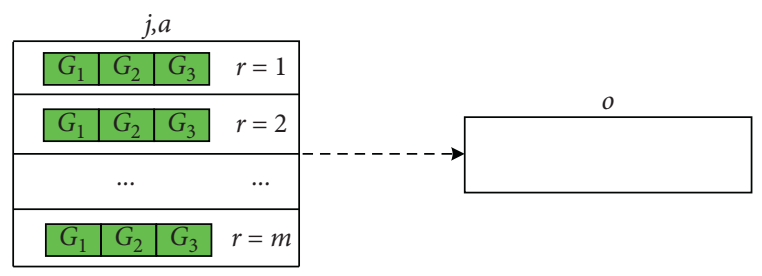

(b)

FiguRE 4: Intersection lane genome.

The set of the fixed phase combination time sequence of the lane in all directions of the entrance section of the intersection when a fixed gene is expressed is as follows:

$$
\mathfrak{R}_{P_{\Phi_{x}}}(k)=\left\{R_{I I I}^{\left(P_{\Phi_{x}}\right)}(k)\right\}_{I I I=1,2, \ldots, \varsigma} .
$$

From formulas (5)-(7), the solution space of lane gene, phase, and phase sequence relationship of intersection is obtained, as shown in Figure 5.

The solution space shown in Figure 5 is described as a feasible solution $g_{j, a, o}(k)$ found in the solution space of formula (4) of the sampling period $k$. The feasible solution can be applied to formula (4) to adjust the time and space variables of green light. Formula (4) is used to adjust the time and space variables of green light.

$$
\begin{aligned}
& X_{x}=\sum_{I=1}^{\varepsilon} \sum_{I I=1}^{\sigma} \sum_{I I I=1}^{\varsigma} \aleph_{I, I I, I I I}, \\
& \text { s.t. } F_{I}^{(x)}(k) \longrightarrow O_{I I}^{\left(\Phi_{x}\right)}(k) \longrightarrow R_{I I I}^{\left(P_{\Phi_{x}}\right)}(k),
\end{aligned}
$$

where $\omega_{j, a}^{X_{x}}(k)$ is to find a feasible solution in the constrained solution space of \{gene, phase, phase sequence\} within 


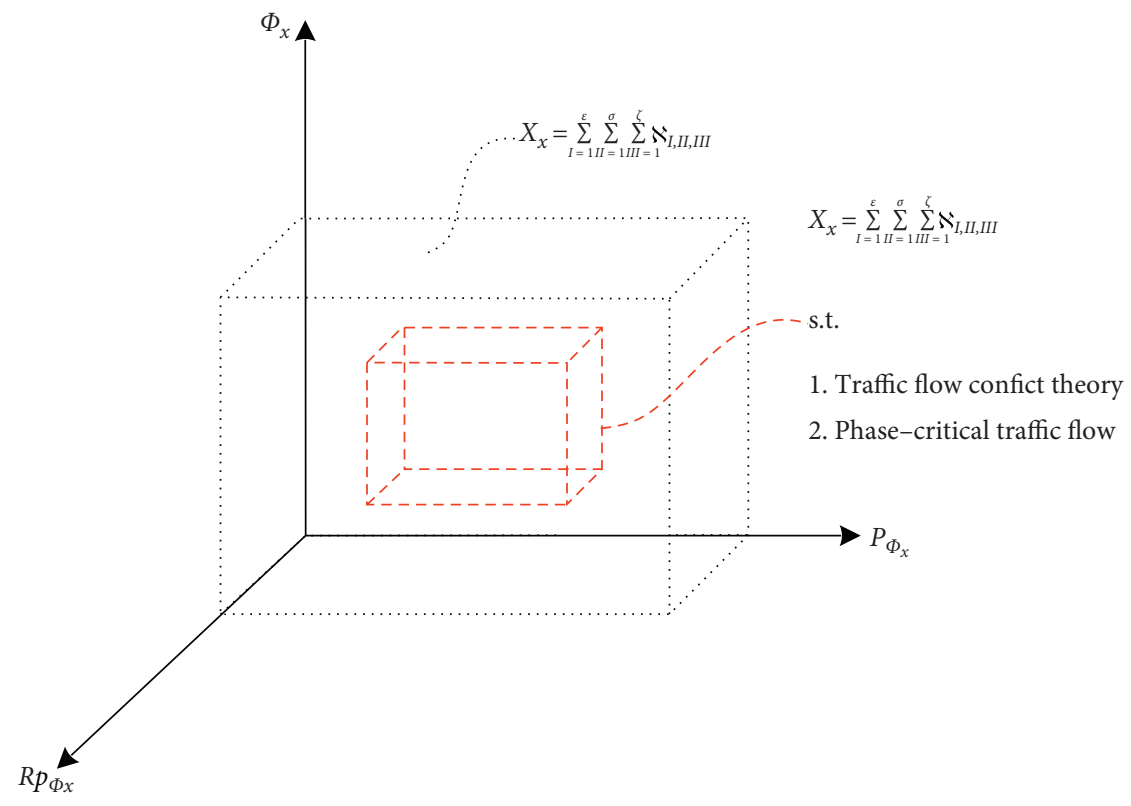

FIGURE 5: Solution space.

period $k$ and the number of phases belonging to road $j, a$ in the phase combination can be obtained, as shown in the following formula:

$$
\omega_{j, a}^{X_{x}}(k)=\left\{X_{x}: O_{I I}^{\left(\Phi_{x}\right)}(k) \subset j \mid F_{I}^{(x)}(k), R_{I I I}^{\left(P_{\Phi_{x}}\right)}(k)\right\} .
$$

In the formula, $o$ of the green time $g_{j, a, o}(k)$ and the spatial variable $\Gamma_{j, a}(k)$ are related to the number of phases, so they can be represented by $\omega_{j, a}^{X_{x}}(k) \cdot \min \left\{\omega_{j, a}(k), \omega_{j, a}^{X_{x}}(k)\right\}$ represents that the number of upstream and downstream links is not the same as the number of phases. The reason is that $\left\{\begin{array}{l}\omega_{j, a}(k) \geq 1 \\ \omega_{j, a}(k) \geq 0\end{array}\right.$, and the upstream and downstream links cannot be disconnected, but the phases cannot be subordinate to the phase sequence within the sampling period $k$.

3.2. Speed Control Model. To realize the study of discrete state and speed control of urban road traffic flow, the basic road traffic flow operation condition is described, as shown in Figure 6.

It is described as follows: the current traffic flow density of road $j$ is equal to the sum of the traffic flow density of road $j$ and the change in traffic flow density of road $j, b$ in the previous time interval.

$$
\rho_{j, b}(k+1)=\rho_{j, b}(k)+\frac{T}{L_{j, b} \cdot \lambda_{j, b}}\left[q_{j, b, \text { in }}(k)-q_{j, b, \text { out }}(k)\right],
$$

where road $j, b$ is used as an example. $q_{j, b, \text { in }}(k)$ is the input flow of road $j, b$ in period $k . q_{j, b \text {,out }}(k)$ is the output flow of road $j, b$ in period $k . v_{j, b}(k)$ is the average speed of traffic flow on road $j, b$ in period $k$. $Q$ is the traffic flow density of road $j, b$ in period $k . L_{j, b}$ is the length of road $j, b$.

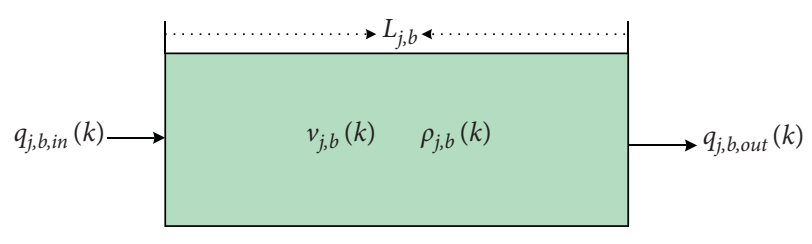

FIGURE 6: Speed control model.

The relationship between dynamic velocity and density parameter and expected velocity of road $j, b$ is constructed to describe the physical characteristics of traffic flow of road $j, b$ according to formula (10). In the model, the dynamic velocity value in the $k+1$ sampling interval is equal to the deviation between the average vehicle velocity in the $k$ sampling interval and the expected driver velocity $V\left[\rho_{j, b}(k)\right]:$

$$
v_{j, b}(k+1)=v_{j, b}(k)+\frac{T}{\tau}\left\{V\left[\rho_{j, b}(k)\right]-v_{j, b}(k)\right\},
$$

where $\tau, v$ is the model parameter and the expected velocity is

$$
V\left[\rho_{j, b}(k)\right]=v_{j, b, \text { free }} \cdot \exp \left[-\frac{1}{\alpha_{j, b}}\left(\frac{\rho_{j, b}(k)}{\rho_{j, b, \text { crit }}}\right)^{\alpha_{j, b}}\right],
$$

where $v_{j, b \text { free }}$ is the free flow velocity of road $j, b, \alpha_{j, b}$ is the model parameter, and $\rho_{j, b, \text { crit }}$ is the critical density of road $j, b$.

3.3. Active Control Model of Intersection. The active traffic control model of the intersection is composed of the temporal and spatial resource dynamic allocation model and the speed control model.

Firstly, the store and forward form of the active traffic control model at intersections is as follows: 


$$
\left\{\begin{array}{l}
q_{j, b, \text { out }}(k)=q_{j, b, \text { in }}(k)-L_{j, b} \cdot \rho_{j, b}(k), \\
n_{j, a}(k+1)=n_{j, a}(k)+q_{j, a, \text { in }}(k)-q_{j, a, \text { out }}(k),
\end{array}\right.
$$

where set $q_{j, b, \text { out }}(k)=q_{j, a, \text { in }}(k)$, and formula (1) is transformed into

$$
n_{j, a}(k+1)=n_{j, a}(k)+q_{j, b, \text { in }}(k)-L_{j, b} \cdot \rho_{j, b}(k)-q_{j, a, \text { out }}(k) .
$$

The complete form of the active traffic control model at the intersection is obtained from formulas (4) and (10):

$$
n_{j, a}(k+1)=n_{j, a}(k)-V(k)-G(k) .
$$

Make

$$
\left\{\begin{array}{l}
V(k)=L_{j, b} \cdot \rho_{j, b}(k), \\
G(k)=q_{j, a, \text { in }}(k)-\sum_{o=0}^{\min \left\{n_{j, a}(k), n_{j, a}^{\mathrm{X} x}(k)\right\}}{\omega_{j, a, o}}(k) \cdot S_{j, a} \cdot g_{j, a, o}(k) .
\end{array}\right.
$$

Put $v(k)=v_{\text {crit }} \cdot \ln \left(\rho_{\text {jam }} / \rho(k)\right)$ into $V(k)$ to get $V(k)=L_{j, b} \cdot \exp \left[-\left(v_{j, b}(k) / v_{j, b, \text { crit }}\right)\right]$. Put it into formula (16) to get

$$
\left\{\begin{array}{l}
V(k)=L_{j, b} \cdot \exp \left[-\frac{v_{j, b}(k)}{v_{j, b, \text { crit }}}\right], \\
G(k)=q_{j, a, \text { in }}(k)-\sum_{o=0}^{\min \left\{n_{j, a}(k), n_{j, a}^{\mathrm{X} x}(k)\right\}} \omega_{j, a, o}(k) \cdot S_{j, a} \cdot g_{j, a, o}(k) .
\end{array}\right.
$$

\section{Optimization Method}

The active control method of the intersection is designed to meet the characteristics of the mixed scene and the requirements of the control goal. Firstly, the current scene of the intersection needs to be accurately identified. Secondly, the objective function is determined based on the identified scene, and then the matching control strategy will be selected. Thirdly, it is necessary to judge whether the capacity coefficient exceeds the critical value when performing phase control. If the critical value is exceeded, boundary control will be activated. Finally, it is necessary to judge whether the capacity exceeds the critical value when performing boundary control. If the critical value is exceeded, lane control will be activated. The active control of the intersection is realized by a two-layer optimization algorithm, as shown in Figure 7.

4.1. Objective Function Set. The mixed scene characteristics of the intersection determine the difference of the control objective function. Considering the unity of objective function design, the lower control objective of the two-layer optimization method is as follows:

$$
\begin{aligned}
& \min \left(\alpha J_{\mathrm{TTS}}+\beta J_{\mathrm{STS}}\right), \\
& \text { s.t. } \alpha+\beta=1,
\end{aligned}
$$

where $J_{\text {TTS }}$ represents the travel time of all vehicles at the intersection; $J_{\text {STS }}$ represents the travel time of a special vehicle.

The choice of weight coefficient is determined by the scene of the intersection. Here are a few examples:

(1) When the scene at the intersection is a common scene, it is to minimize the total travel time of vehicles passing at the intersection as the objective function.

(2) When the scene at the intersection is a special scene of emergency vehicles, it is to minimize the travel time for emergency vehicles to pass through the intersection as the objective function.

(3) When the scene at the intersection is a special scene of bus priority vehicles, it is to minimize the travel time of bus priority vehicles and all other passing vehicles at the intersection as the objective function.

The difference in starting order and conditions between speed control and lane control should be considered. The upper control objectives of the two-layer optimization method are characterized by time continuity and form unity. The upper control objective of the two-layer optimization method is as follows:

$$
\max _{t \rightarrow \Delta} J_{s}(t)
$$

Make

$$
\left\{\begin{array}{l}
\lim _{t \longrightarrow \tau}|| J_{s}(t)\left|-\overline{J_{s}}\right|=\varepsilon, \\
\lim _{t \longrightarrow \pi}|| J_{s}(t)\left|-\overline{J_{s}}\right|=\varepsilon,
\end{array} \quad \text { s.t. } t \longrightarrow \tau \longrightarrow \pi,\right.
$$

where $\overline{J_{s}}=0$ represents the critical value of the capacity coefficient; $\varepsilon \longrightarrow 0$ is the minimum.

4.2. Control of Dynamic Phase and Virtual Phase. Dynamic phase control adopts the phase control algorithm of model predictive control. The particularity of bus priority and emergency control is considered. The dynamic phase control algorithm is extended and a control algorithm combining dynamic phase and virtual phase is adopted. It is an extension of the length of the control chain in the dynamic phase control algorithm, where the last phase of the predictive control chain is defined as a virtual phase to respond to the control requirements in special scenarios. Among them, the virtual phase description does not specify a specific phase type. When a special scene requires its expression, the best phase is selected in the intersection phase set to respond.

A control algorithm combining the dynamic phase and virtual phase is shown in Figure 8 . When a special scene appears, the virtual phase is activated. At this time, its input is the traffic and queuing at the intersection when a certain phase is executed in the sampling period $k$. Then, the 


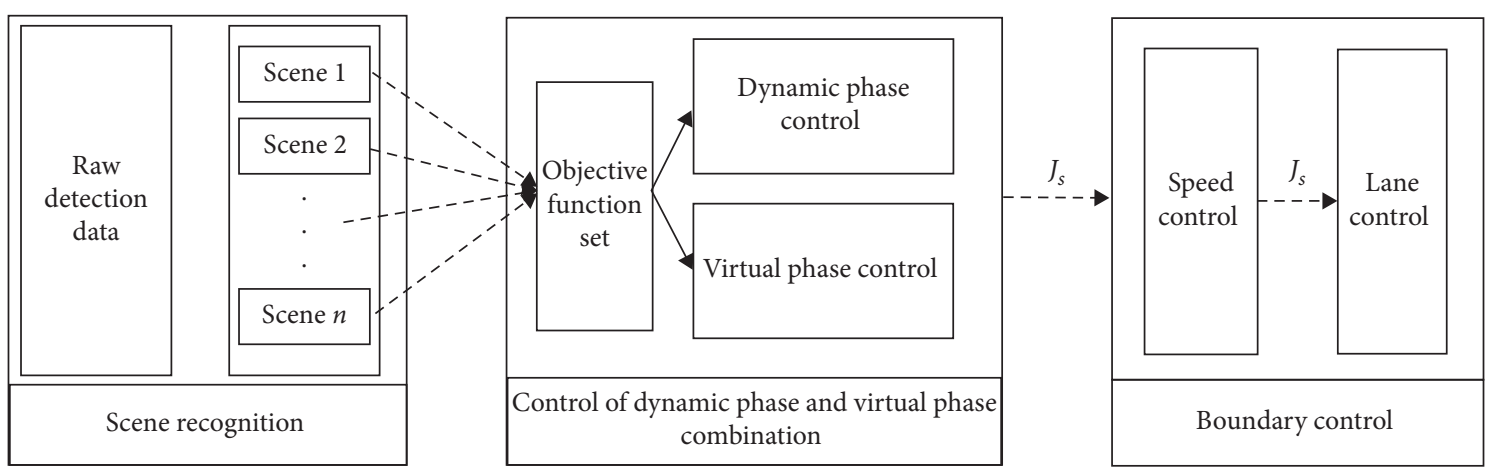

FIGURE 7: Structure of active control at the intersection.

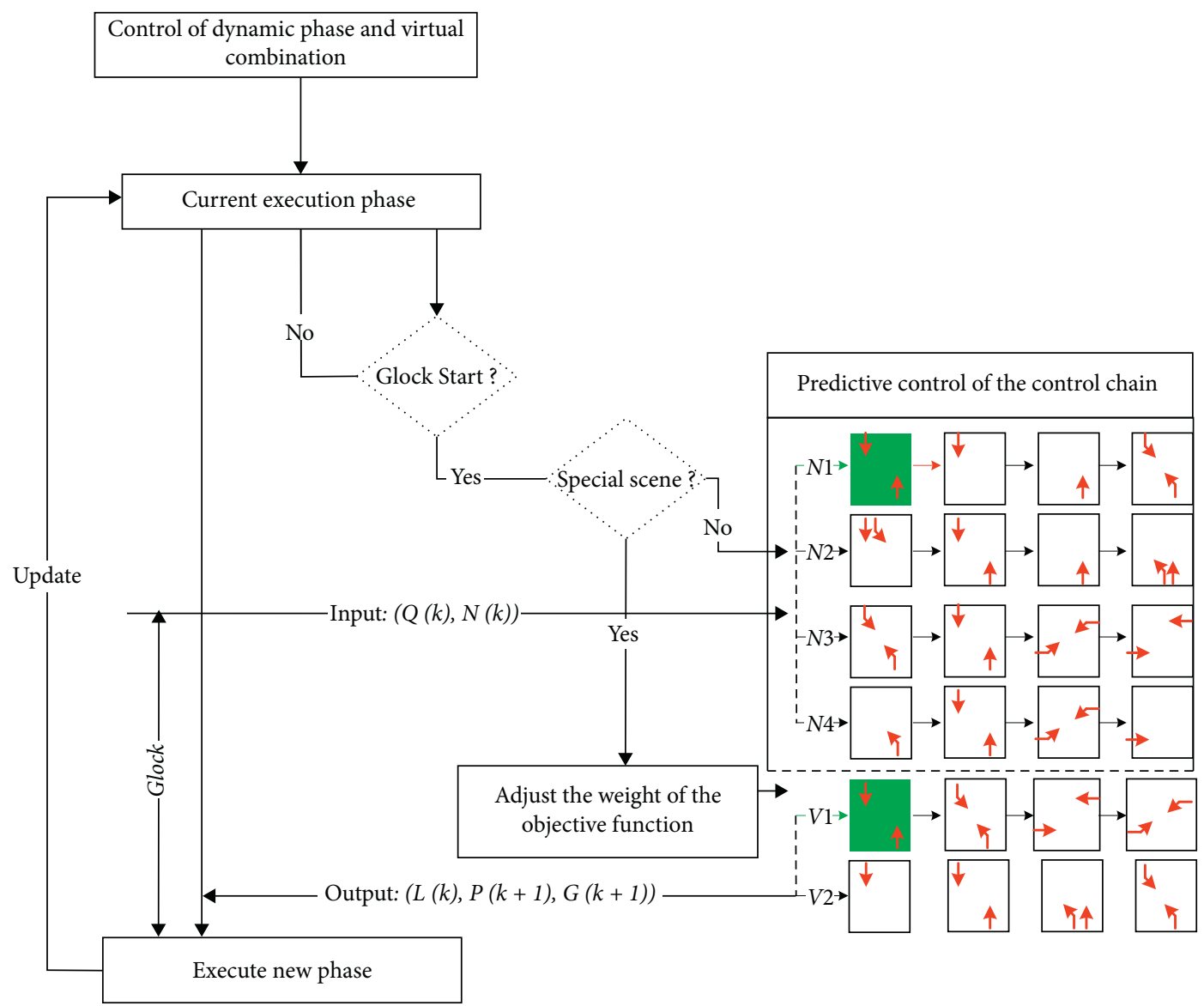

FIGURE 8: The control algorithm of dynamic phase and virtual phase combination.

candidate phase to be executed in the next step is to search for $n$ phases that match the currently executed phase in the solution space. Finally, the generation of the control chain is to select $m$ consecutively executed phases in each candidate phase. $J=\min \left(\alpha J_{\mathrm{TTS}}+\beta J_{\mathrm{STS}}\right)$, s.t. $\alpha+\beta=1$ is taken as the objective function. According to the adjustment weight value, the form of the objective function is changed. The GA algorithm is used to execute $n$ control chains and rank the resulting $J$ of $n$ control chains. The first phase of the control chain with the minimum $J$ obtained as a result is regarded as the next execution phase of the current phase, and the obtained interval time, phase, and green light time are used as output.

4.3. Two-Layer Optimization 1: Speed Control. As shown in Figure 9, the combination of speed control and dynamic phase and its extended control methods form the internal and external control areas of the intersection with boundary characteristics. They are called speed control zones and lane 


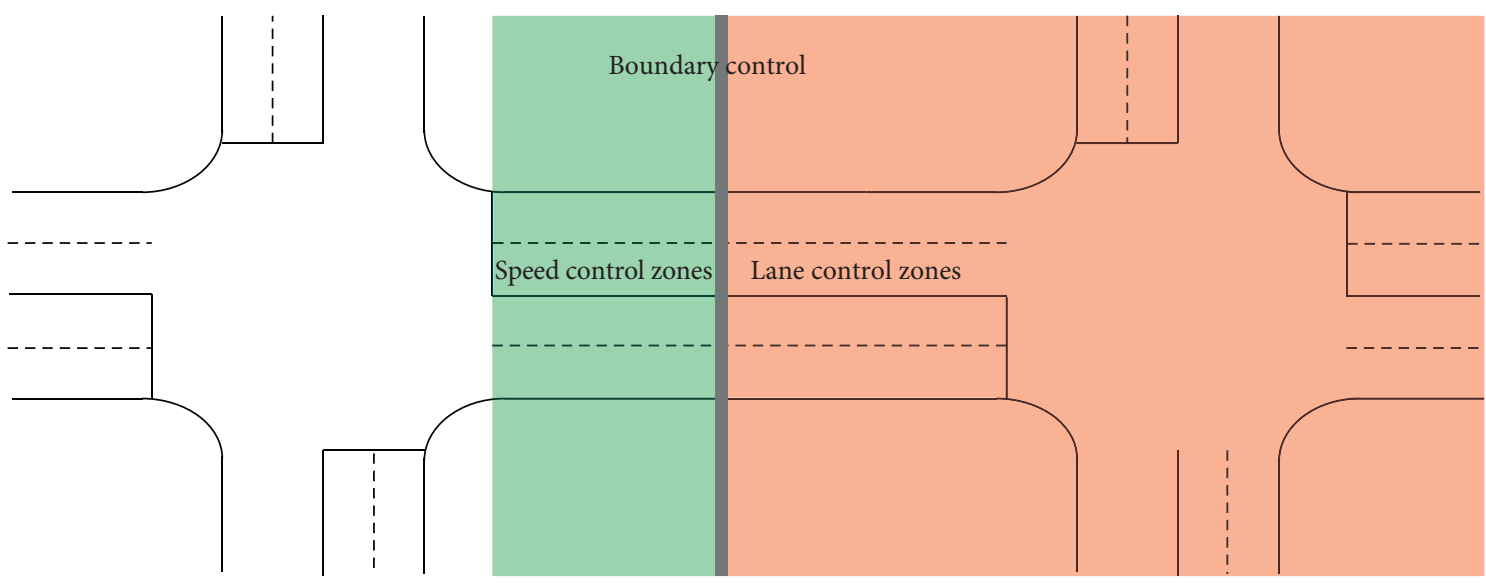

Figure 9: Boundary control.

control zones. The boundary control idea is adopted. It determines whether to start speed control by judging the change of $J_{S}$. It can achieve the purpose of reducing the number of vehicles in the control area by suppressing the input flow.

Two-level optimization 1 uses a two-level programming algorithm. The basis of the upper-level planning is the speed control of the speed control area in Figure 9. When the $J_{S}$ control area reaches the critical value, it means that the traffic capacity has reached the limit. At this time, formula (21) is used as the objective function of the upper-level planning:

$$
J_{\text {up }}(t)=\max _{t \longrightarrow \Delta} J_{s}(t)=\max _{t \longrightarrow \Delta}\left(J_{M}(t)-J_{N}(t)\right) .
$$

The specific form of upper-level planning constraints and capacity coefficient $J_{S}$ is as follows:

$$
\left\{\begin{array}{l}
J_{M}=\frac{\sum_{j=1}^{n} S_{j, a} \cdot g_{j, a, o}(k)}{\sum_{j=1}^{n}\left(S_{j, a} \cdot g_{j, a, o}(k)+\left(\Delta n_{j, a}(k) / \overline{n_{j, a}}\right)\right)}, \\
J_{N}=\frac{\sum_{i=1}^{n}\left(\Delta n_{j, a}(k) / \overline{n_{j, a}}\right)}{\sum_{j=1}^{n}\left(S_{j, a} \cdot g_{j, a, o}(k)+\left(\Delta n_{j, a}(k) / \overline{n_{j, a}}\right)\right)}, \\
\Delta n_{j, a}(k)=n_{j, a}(k+1)-n_{j, a}(k)=-V(k) \\
-G(k) \lim _{t \longrightarrow \tau}|| J_{s}(k)\left|-\overline{J_{s}}\right|=\varepsilon .
\end{array}\right.
$$

The objective function and constraint conditions of the lower-level planning adopt formula (21). The lower-level planning solution of the two-level optimization 1 uses a combination of dynamic phase and virtual phase control algorithm.

4.4. Two-Layer Optimization 2: Lane Control. Two-layer optimization 2 uses a two-level planning algorithm composed of dynamic phase and virtual phase control and lane control. The objective functions of the upper and lower layers need to follow the requirements of the objective function set above, and the time when the lane control is activated is determined by $\left\{\begin{array}{l}\lim _{t \rightarrow \tau}|| J_{s}(t)\left|-\overline{J_{s}}\right|=\varepsilon, \\ \lim _{t \rightarrow \pi}|| J_{s}(t)\left|-\overline{J_{s}}\right|=\varepsilon,\end{array}\right.$ s.t. $t \longrightarrow \tau \longrightarrow \pi$.

4.5. Algorithm for Active Control of Intersection. The algorithm flow is shown in Figure 10 and the algorithm steps are shown in Algorithm 1.

\section{Simulation Experiment}

5.1. Simulation Environment. The six scenarios in the paper are designed for comparison with traditional methods that include undersaturation, oversaturation, undersaturation and bus priority, oversaturation and bus priority, undersaturation and emergency vehicles, and oversaturation and emergency vehicles, which are divided into three groups for experiments. The self-developed Open Simulation Platform (OSP) is used for experiments. The simulated road network is shown in Figure 11, and the specific parameter settings are shown in Table 1.

A further explanation for the experiment is as follows:

Traditional control methods are generally pertinent; that is, different scenarios will adopt adaptive control methods.

(1) For the reasonableness of comparison, in the first set of \{undersaturated and oversaturated\} two scenarios, the comparison is with timing control; in the second set of \{undersaturated and bus priority, oversaturated and bus priority\} two scenarios, the comparison is with traditional bus priority control [27] (using phase green light extension and red light shortening); in the third set of (undersaturated and emergency vehicles, oversaturated and emergency vehicles\} two scenarios, the comparison is with traditional emergency vehicle control [28] (using trigger response; that is, when a traffic request is detected, the phase is locked or jumped immediately).

(2) In the simulation, the detection points are used to replace the commonly used RFID detectors in practice, and the detection points are, respectively, 


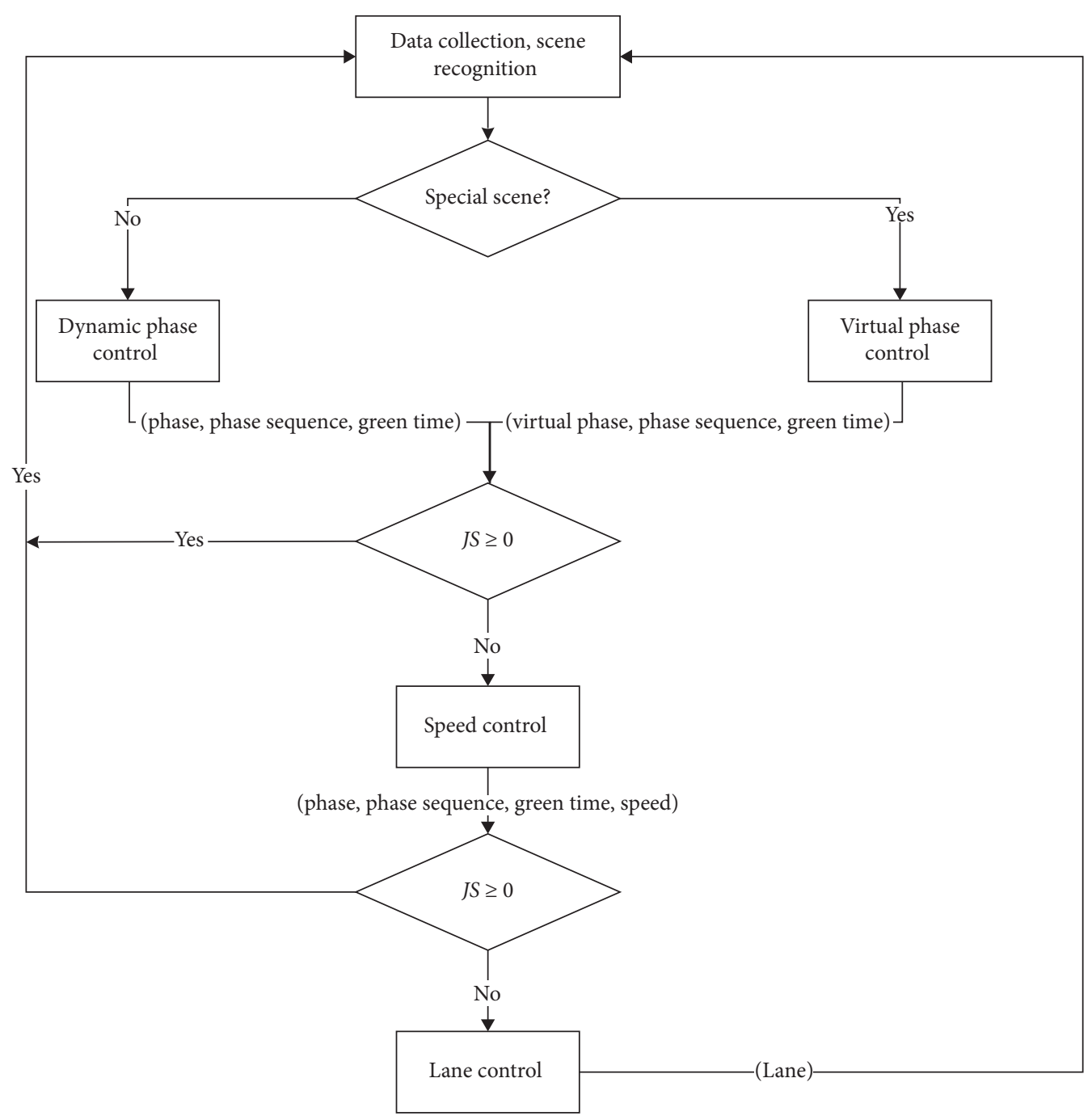

FIGURE 10: Algorithm flow of active control of intersection.

Stage 1: start

Step 1.1: data collection and scene recognition

Step 1.2: it judges whether the scene recognized by Step 1.1 is a special scene. If it is, the dynamic phase and virtual phase combination control are activated at the current intersection. First, the virtual phase is activated, and the best phase suitable for the scene is selected for control in the phase set of the intersection. The imaginary phase can be the continuation of the current phase or any phase in the phase set. The optimization of the objective function corresponding to the scene determines whether it is the best phase, and outputs \{virtual phase, phase sequence, green light time\}. Otherwise, go to Step 1.3.

Step 1.3: the dynamic phase control is activated and \{phase, phase sequence, green time\} is output.

Step 1.4: go to stage 2 .

Stage 2: two-layer optimization 1

Step 2.1: if $J_{S} \geq 0$ exists for $n$ consecutive sampling periods, go to Step 1.1. Otherwise, go to Step 2.2.

Step 2.2: if there is $J_{S}=0$ for $n$ consecutive sampling periods, the speed control is activated and the output \{phase/virtual phase, phase sequence, green time, speed\}. If there is $J_{S} \geq 0$ for $n$ consecutive sampling periods, if it is, go to Step 1.1. Otherwise, go to Step 2.3. Step 2.3: go to stage 3;

Stage 3: two-layer optimization 2

Step 3.1: if $J_{S}=0$ exists for $n$ consecutive sampling periods, lane control is activated. The lane attributes, phase set, and control chain of the intersection are updated, and output \{phase/virtual phase, phase sequence, green time, speed, lane\}, and Go to Stage 1. 


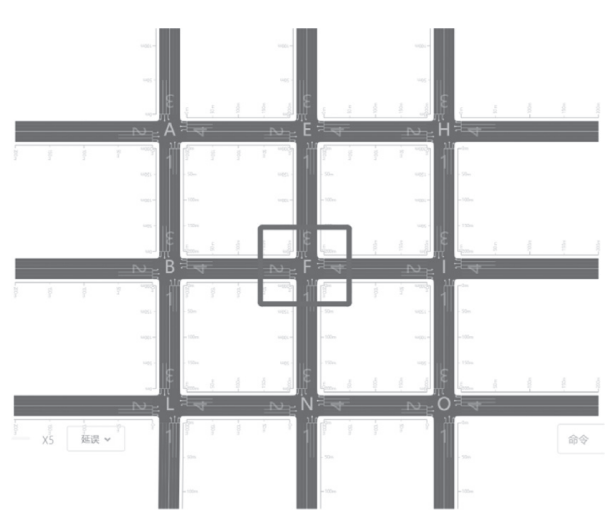

FIGURE 11: Road simulation of OSP.

TABLE 1: Basic parameters of the simulation.

\begin{tabular}{|c|c|}
\hline Parameter & Content \\
\hline Road & $\begin{array}{l}\text { The road network is } 3 * 3 \text { intersections; the length of the intersection connecting section is } 470 \mathrm{~m}-490 \mathrm{~m} \text {; the } \\
\text { section uses two-way } 4 \text { lanes, and the channelization is set at } 30 \text { meters at the entrance of the intersection. The } \\
\text { width is } 3.5 \mathrm{~m}\end{array}$ \\
\hline Vehicle & $\begin{array}{c}\text { The speed distribution is }[20 \mathrm{~km} / \mathrm{h}, 60 \mathrm{~km} / \mathrm{h}] \text {, the ratio of vehicle types is } 1: 99 \text { (large cars: small cars), and the } \\
\text { ratio of buses to emergency vehicles is } 99: 1 \text { in the large car }\end{array}$ \\
\hline Flow & $\begin{array}{l}\text { Initial: the flow of stage } 1 \text { is } 1000 \mathrm{v} / \mathrm{h} \text {; the flow of stage } 2 \text { is } 500 \mathrm{v} / \mathrm{h} \text {; the flow of stage } 3 \text { is } 1000 \mathrm{v} / \mathrm{h} \text {; the flow of } \\
\text { stage } 4 \text { is } 500 \mathrm{v} / \mathrm{h} \text {. Every } 3600 \mathrm{~s} \text { multiply the flow of each stage by the coefficient of change } x, 0.5<x<2\end{array}$ \\
\hline $\begin{array}{l}\text { Intersection and signal } \\
\text { control }\end{array}$ & $\begin{array}{l}\text { The intersections are numbered A, B, E, F, H, I, L, N, and O, the number of which that is controlled in this } \\
\text { chapter is F; except for intersection F, all other intersections are controlled by 4-stage signal lights. The stages } \\
\text { are stage1: north-south straight; stage2: north-south left; stage } 3 \text { : east-west straight; stage4: turn left from east } \\
\text { to west; straight-right vehicles and straight-going vehicles are released at the same time; the signal control } \\
\text { period is } 120 \mathrm{~s} \text {; the interval time is } 3 \mathrm{~s} \text { for yellow light and } 2 \mathrm{~s} \text { for all red; Tstage } 1 \text { is } 35 \mathrm{~s} \text {, Tstage } 2 \text { is } 15 \mathrm{~s} \text {; Tstage } 3 \text { is } \\
\qquad 35 \mathrm{~s} \text {; and Tstage } 4 \text { is } 15 \mathrm{~s}\end{array}$ \\
\hline Scenarios & $\begin{array}{l}\text { Undersaturation, oversaturation, undersaturation and bus priority, oversaturation and bus priority, } \\
\text { undersaturation and emergency vehicles, and oversaturation and emergency vehicles }\end{array}$ \\
\hline Data collection & $\begin{array}{l}\text { Collect the road section number, lane number, whole road section density, flow, and vehicle speed. The } \\
\text { acquisition interval is } 600 \mathrm{~s}\end{array}$ \\
\hline Evaluation parameters & The average number of stops and average delay time \\
\hline
\end{tabular}

arranged at positions about $150 \mathrm{~m}$ away from the parking line.

(3) All buses appearing in the simulation are subject to public transportation priority control, and all emergency vehicles appearing are subject to emergency priority control.

(4) In the simulation, select the objective function parameters $\alpha$ and $\beta$ : in the first set of scenarios \{undersaturated, oversaturated $\}$, set $\alpha=1, \beta=0$; that is, consider the minimum total travel time of vehicles passing through the intersection as the objective function. In the second set of scenarios \{undersaturation and bus priority, oversaturation and bus priority\}, set $\alpha=0, \beta=1$; that is, consider the minimum travel time of bus priority vehicles passing through the intersection as the objective function. In the third set of scenarios \{undersaturated and emergency vehicles, oversaturated and emergency

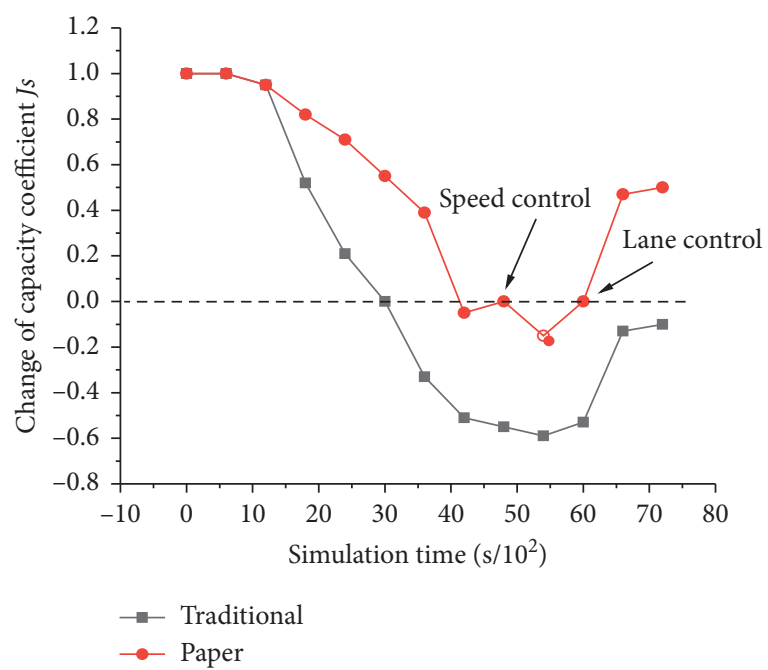

Figure 12: Change of capacity coefficient. 


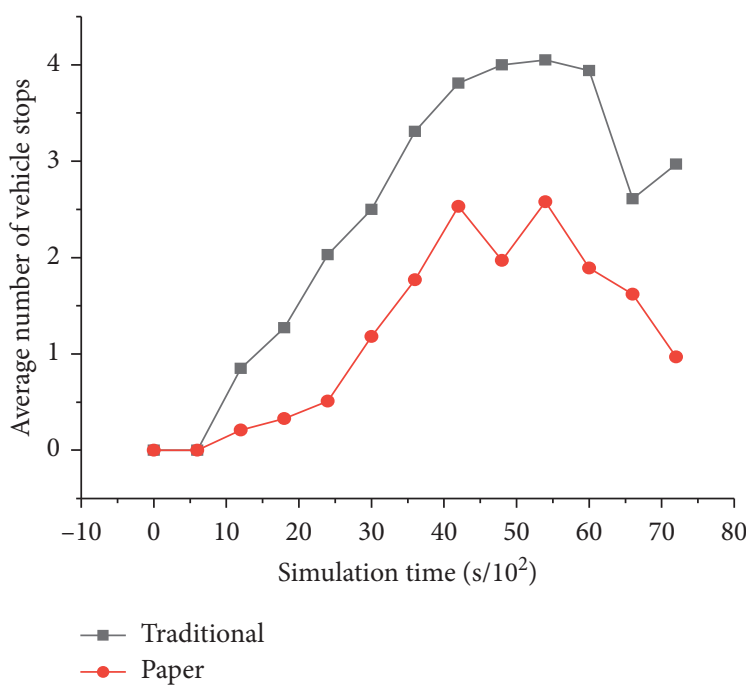

(a)

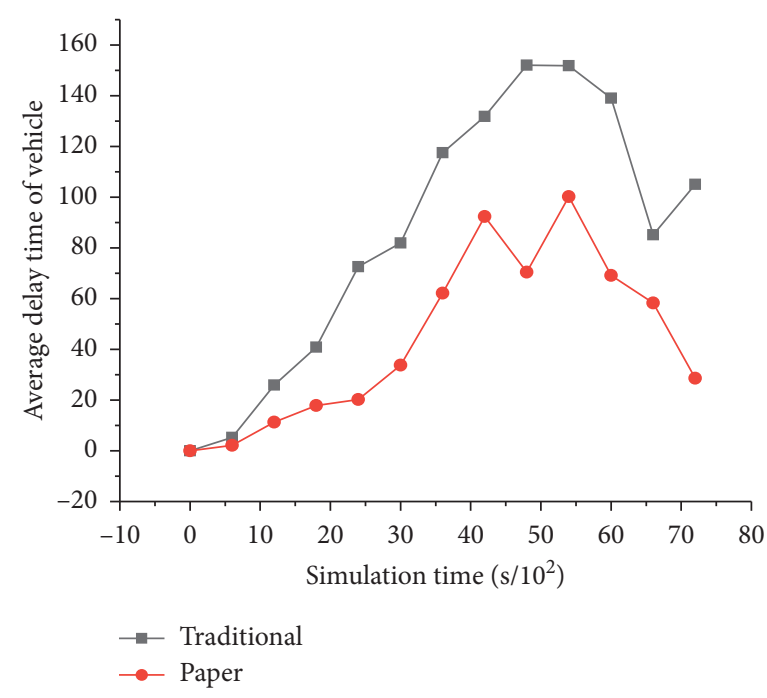

(b)

Figure 13: The contrast of control effect of the first set of scenarios. (a) Average number of vehicle stops at intersections. (b) Average delay time of vehicle at the intersection.

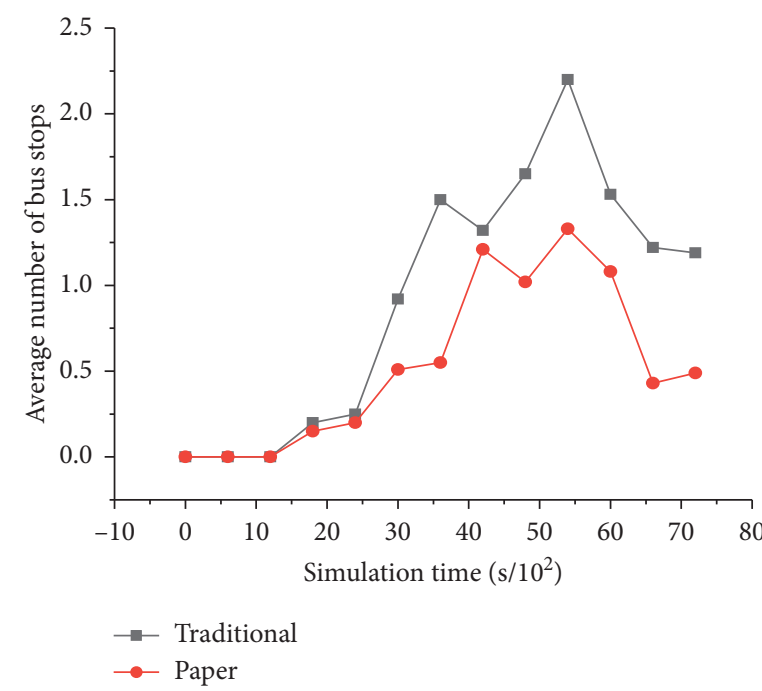

(a)

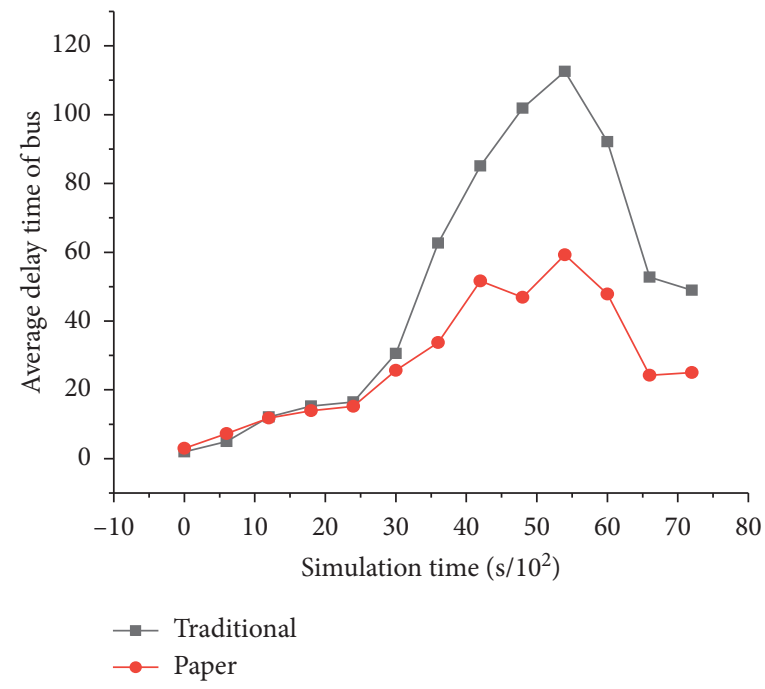

(b)

Figure 14: The contrast of control effect of the second set of scenarios. (a) Average number of bus stops. (b) Average delay time of the bus.

vehicles\}, set $\alpha=0, \beta=1$; that is, consider the minimum travel time of emergency vehicles through the intersection as the objective function (the purpose of choosing the objective function parameters in this way is to be consistent with the traditional comparison of control methods).

\subsection{Analysis Results}

5.2.1. The First Set of Scenes: \{Undersaturated, Oversaturated\}. Figure 12 shows the change of the capacity coefficient $J_{S}$ when the two scenarios included in the first group are compared. It can be seen from Figure 12 that the time to reach the $J_{S}$ threshold is later when using the method in this paper than when using the traditional control method. Arrow 1 (speed control) shown in the figure indicates that $J_{S}<0$, and the speed control is started, but it is found that $J_{S}$ of the next data point is further reduced, indicating that the speed control can no longer control the decline of $J_{S}$ at this time, and then start lane control shown in Figure 12, due to the subsequent improvement of the traffic state and the effect of lane control, makes $J_{S}$ return to the control threshold.

Figures 13(a) and 13(b), respectively, show the average number of stops and average delay time of vehicles passing 


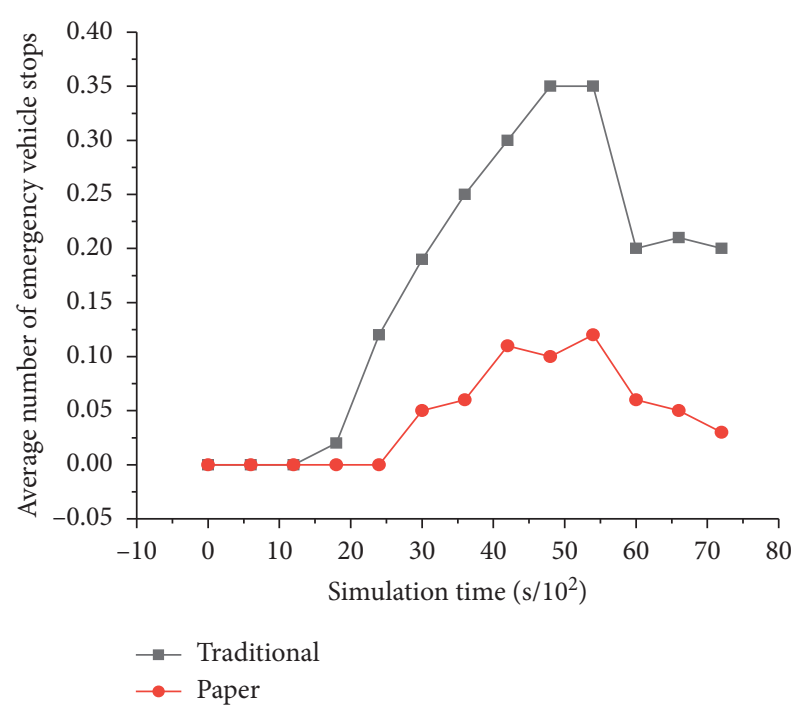

(a)

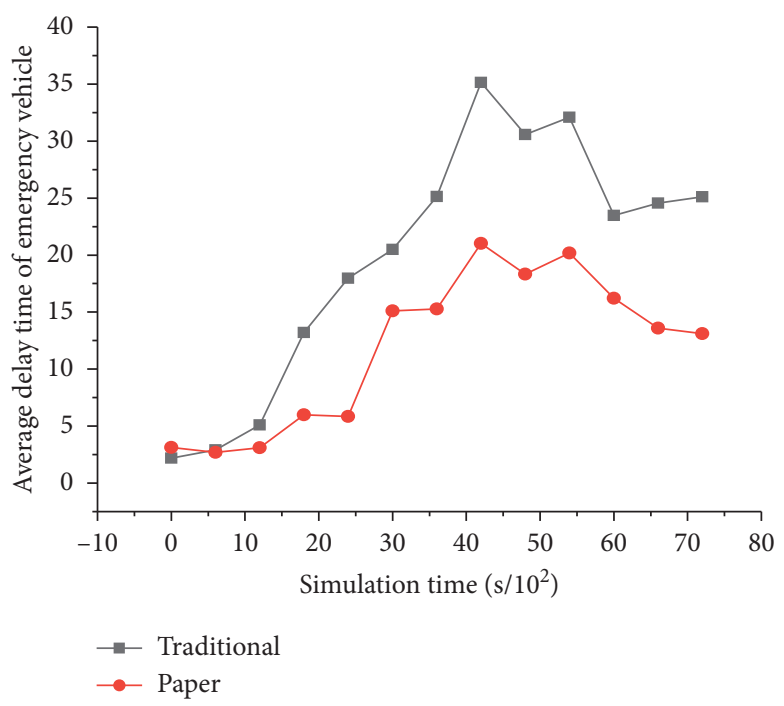

(b)

Figure 15: The contrast of control effect of the third set of scenarios. (a) Average number emergency vehicle stops. (b) Average delay time of emergency vehicle.

TABLE 2: Comparison of simulation results of control methods.

\begin{tabular}{|c|c|c|c|c|}
\hline Experimental scene & Method & Evaluation parameters & Results & Improve \\
\hline \multirow{4}{*}{ The first set of scenes } & Traditional & Average stops & 92.4 & - \\
\hline & Paper & Average delay time & 59.2 & $35.93 \%$ \\
\hline & Traditional & Average stops & 2.41 & - \\
\hline & Paper & Average delay time & 1.64 & $31.80 \%$ \\
\hline \multirow{4}{*}{ The second set of scenes } & Traditional & Average stops & 50.1 & - \\
\hline & Paper & Average delay time & 38.4 & $22.35 \%$ \\
\hline & Traditional & Average stops & 0.79 & - \\
\hline & Paper & Average delay time & 0.58 & $26.58 \%$ \\
\hline \multirow{4}{*}{ The third set of scenes } & Traditional & Average stops & 16.2 & - \\
\hline & Paper & Average delay time & 13.8 & $14.81 \%$ \\
\hline & Traditional & Average stops & 0.17 & - \\
\hline & Paper & Average delay time & 0.09 & $47.06 \%$ \\
\hline
\end{tabular}

through the intersection in the first set of scenarios. It can be seen from Figure 13 that the control effect of the active control method proposed in this article is better than that of the traditional control method in the two scenarios of undersaturation and oversaturation.

\subsubsection{The Second Set of Scenarios: \{Undersaturation and Bus} Priority, Oversaturation and Bus Priority\}. Figures 14(a) and 14(b), respectively, show the average number of stops and average delay time of buses passing through the intersection in the second set of scenarios. It can be seen from Figure 14 that, in the scenario of undersaturation and bus priority, the control effect of the active control method proposed in this article is similar to that of the traditional control method; however, as the traffic flow changes, the scene changes from undersaturation and bus priority to oversaturation and bus priority scenario, and the control effect of the active control method proposed in this paper is better than that of the traditional control method.
5.2.3. The Third Set of Scenes: \{Undersaturated and Emergency Vehicles, Oversaturated and Emergency Vehicles\}. Figures 15(a) and 15(b), respectively, show the average number of stops and average delay time of buses passing through the intersection in the third set of scenarios. It can be seen from Figure 15 that, in the scenario of undersaturated and emergency vehicles, the control effect of the active control method proposed in this article is similar to that of the traditional control method; and in the scenario of oversaturated and emergency vehicle, the control effect of the active control method proposed in this paper is better than that of the traditional control method.

As shown in Table 2, compared with the traditional method, in the first set of scenarios, the average parking delay time at intersections is reduced by $35.93 \% \%$, and the average number of stops is $31.80 \%$; in the second set of scenarios, the average bus parking delay time is reduced by $22.35 \%$, and the average number of parking times is $26.58 \%$; in the third group of scenarios, the average parking delay 


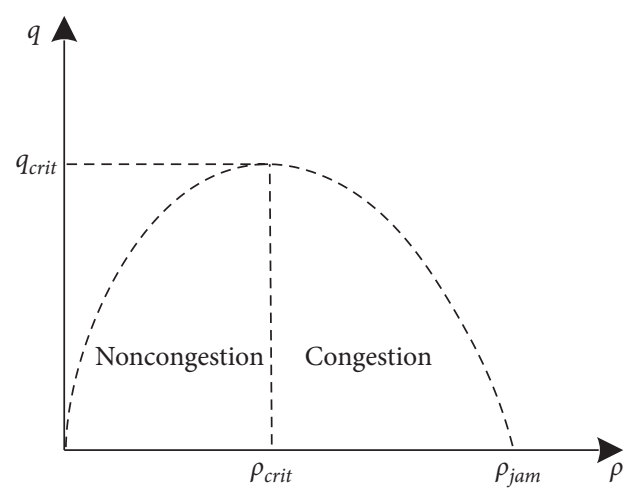

(a)

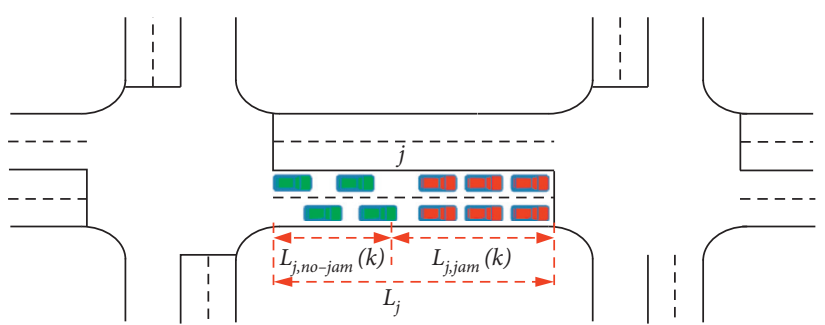

(b)

Figure 16: Second-flow theory. (a) Basic diagram of traffic flow $q \longrightarrow \rho$. (b) Schematic diagram of the second-rate theory.

time of emergency vehicles is reduced by $14.81 \%$, and the average number of parking times is $47.06 \%$. It can be seen that the method in this paper can give suitable control responses for different scenarios, which is very efficient in traffic.

\section{Conclusion Remark and Future Work}

This paper proposes a multivariable active traffic control model (ATCM) based on the vehicle infrastructure cooperative technology. The model increases the control variables from two dimensions to five dimensions and almost contains all the time and space variables of the intersection. It can be imagined that the flexibility of intersection control will be greatly improved, and in particular it can reshape the traffic flow organization and traffic control mode of intersection, which is very beneficial for the future fully automatic driving scene. The d-bilevel optimization method is designed for ATCM. In the first part of the method, boundary control is used to combine speed control with dynamic phase sequence control to provide initial control scheme. In the second part, space-time coordinated control is used to combine lane control with dynamic phase sequence control to provide more powerful control scheme. The traditional simulation platform is difficult to meet the experimental requirements of the paper, so the OSP is adopted. Three contrast scenarios are designed in the simulation. The \{undersaturated and oversaturated\} scenarios are used to verify the congestion mitigation capability of the model, the \{undersaturated and bus priority, oversaturated and bus priority\} scenarios are used to verify the capability of the model for bus priority, and the \{undersaturated and emergency vehicles, oversaturated and emergency vehicles\} scenarios are used to simulate the capability of the model to deal with the sudden demand of characteristic vehicles. The experimental results show that the proposed method is superior to the traditional method in reducing the average delay time and the average number of stops. It can be seen that ATCM has great potential to adapt to more complex control requirements of the intersection. In the future work, this method will be extended to hybrid driving traffic control, fully automatic driving traffic control, and other fields.

\section{Appendix}

\section{A.1. Model Constraints}

A.1.1. Division of Lane Control Area and Vehicle Speed Control Area Based on the Second-Flow Theory. A type of typical urban road section is modeled. According to the basic diagram of traffic flow $q \longrightarrow \rho$, it can be seen that when the density of the road section is the optimal density, the flow reaches the maximum value. At this time, the traffic flow can be divided into two states, noncongested flow and congested flow, as shown in Figure 16.

Single-lane model based on second-flow theory is as follows:

$$
\left\{\begin{array}{l}
n_{j, \text { init }}+q_{j, \text { in }}(k)=q_{j, \text { out }}(k)+n_{j}(k), \\
n_{j}(k)=\rho_{\text {jam }} \cdot L_{j, \text { jam }}(k)+\rho_{\text {crit }}\left(L_{j}-L_{j, \text { jam }}(k)\right) .
\end{array}\right.
$$

From formula (A.1),

$$
L_{j, \mathrm{jam}}(k)=\frac{n_{j}(k)+q_{j, \text { in }}(k)-q_{j, \mathrm{out}}(k)-\rho_{\text {crit }} \cdot L_{j}}{\left(\rho_{\mathrm{jam}}-\rho_{\text {crit }}\right)} .
$$

Formula (A.3) is extended to obtain the average equivalent queue length model of multilane road segments because it takes into account the multisegment characteristics of urban roads.

$$
L_{j, \text { jam }}(k)=\frac{n_{j}(k)+q_{j \text {,in }}(k)-q_{j, \text { out }}(k)-\rho_{\text {crit }} \cdot L_{j} \cdot \lambda_{j}}{\lambda_{j}\left(\rho_{\text {jam }}-\rho_{\text {crit }}\right)} .
$$

The road section $j$ is divided into congested flow area and noncongested flow area according to formula (A.3). The length of the congested flow area is $L_{j \text {,jam }}(k)$, and the length of the uncongested flow is $L_{j \text {,no-jam }}(k)=L_{j}-L_{j \text {,jam }}(k)$. Consider the road section of the noncongested flow as the vehicle speed control area and the road section of the 
congested flow as the lane control area. Considering the regularity of the macroscopic traffic flow, the length of the lane control area is $L_{j, a}=\overline{L_{j, j a m}}$ and the length of the vehicle speed control area is $L_{j, b}=\overline{L_{j, \text { no-jam }}}$; and the division of road section length does not change with traffic flow.

A.1.2. Lane Control Area and Speed Control Area Range Requirements. In order to ensure the safety and efficiency of vehicles, the following requirements are given for the length $L_{j, a}$ of lane control area and the length $L_{j, b}$ of speed control area:

$$
\left\{\begin{array}{l}
L_{j, a} \geq L_{j, a \min }, \\
L_{j, b} \geq L_{j, b, \min }, \\
L_{j}=L_{j, a}+L_{j, b},
\end{array}\right.
$$

where

(1) $L_{j, a, \min }=\overline{L_{j, j \mathrm{am}}}+\ell$, and $\ell$ is the adjusted value. The average queue length $L_{j, a \text {, min }}$ is obtained based on experience or historical data and is generally $150 \mathrm{~m}-200 \mathrm{~m}$.

(2) $L_{j, b, \min }=\left|\left(1 / 2 \psi_{j, b}\right)\left(v_{j, b, \text { free }}^{2}-v_{j, b, v s l \text { min }}^{2}\right)\right|$.

\section{Data Availability}

The actual verification and test of the system are supported by Weifang Traffic Police Department in Shandong Province and the Beijing Shunyi District Traffic Police Department. The data provided is for use in this paper only because it involves commercial and government requirements for the use of data.

\section{Conflicts of Interest}

The authors declare that there are no conflicts of interest.

\section{Authors' Contributions}

Conceptualization was performed by Li-li Zhang and Qi Zhao; methodology was prepared by Li-li Zhang and Fang Wang; resources were provided by Li Wang; original draft preparatio was carried out by Li-li Zhang and Yadongyang Zhu; review and editing were done by Qi Zhao and Ling-yu Zhang; visualization was conducted by Ling-yu Zhang; supervision was performed by Li Wang.

\section{Acknowledgments}

This research was supported by grants from the Beijing $\mathrm{Mu}$ nicipal Great Wall Scholar Program (CIT\&TCD20190304), the National Key R\&D Program of China (2017YFC0821102 and 2017YFC0822504), the 2021-2023 Young Talents Promotion Project of Beijing Association for Science and Technology, Cross Research Project of Beijing Institute of Petrochemical Technology, General Project of Science and Technology Plan of Beijing Municipal Education Commission (KM201910017006 and KM202010017011) and the Beijing Municipal Natural Science Foundation (4214070).

\section{References}

[1] P. B. Hunt, D. I. Robertson, R. D. Bretherton, and M. C. Royle, "The SCOOT on-line traffic signal optimization technique," Traffic Engineering Control, vol. 4, pp. 142-149, 1982.

[2] J. Y. K. Luk, "Two traffic-responsive area traffic control methods: SCAT and SCOOT," Traffic Engineering Control, vol. 25, no. 1, pp. 100-109, 1984.

[3] P. Lowrie, A Traffic Responsive Method of Controlling Urban Traffic, Roads and Traffic Authority, SCATS, Sydney, Australia, 1990.

[4] P. Lowrie, A Traffic Responsive Method of Controlling Urban Traffic, Roads and Traffic Authority, SCATS, Sydney, Australia, 1992.

[5] G. Rafiq, B. Talha, M. Patzold et al., "What?s new in intelligent transportation systems?: an overview of European projects and initiatives," IEEE Vehicular Technology Magazine, vol. 8, no. 4, pp. 45-69, 2013.

[6] D. Green, P. Bennett, C. Han et al., "“'Cooperative intelligent transport systems (C-ITS) - an overview of the ARRB/Austroads C-ITS work program 2010-2013," World Journal of Diabetes, vol. 23, no. 2, pp. 15-28, 2015.

[7] M. Teichmann, M. Weber, M. Zoellner et al., "Multinet: realtime joint semantic reasoning for autonomous driving," in Proceedings of the 2018 IEEE Intelligent Vehicles Symposium, pp. 1013-1020, Changshu, China, June 2018.

[8] J. Wang, J. Liu, and N. Kato, "Networking and communications in autonomous driving: a survey," IEEE Communications Surveys Tutorials, vol. 21, no. 2, pp. 1243-1274, 2018.

[9] M. Sefati, J. Chandiramani, K. Kreiskoether et al., "Towards tactical behaviour planning under uncertainties for automated vehicles in urban scenarios," in Proceedings of the IEEE 20th International Conference on Intelligent Transportation Systems, pp. 1-7, Yokohama, Japan, October 2017.

[10] T. H. Chang and G. Y. Sun, "Modeling and optimization of an oversaturated signalized network," Transportation Research Part B, vol. 38, no. 8, pp. 4687-4707, 2004.

[11] H. Liu, K. N. Balke, and W.-H. Lin, "A reverse causal-effect modeling approach for signal control of an oversaturated intersection," Transportation Research Part C: Emerging Technologies, vol. 16, no. 6, pp. 742-754, 2008.

[12] D. C. Gazis, "Optimum control of a system of oversaturated intersections," Operations Research, vol. 12, no. 6, pp. 815-831, 1964.

[13] M. Papageorgiou, C. Kiakaki, V. Dinopoulou, A. Kotsialos, and W. Yibing, "Review of road traffic control strategies," Proceedings of the IEEE, vol. 91, no. 12, pp. 2043-2067, 2003.

[14] T.-H. Chang and J.-T. Lin, "Optimal signal timing for an oversaturated intersection," Transportation Research Part B: Methodological, vol. 34, no. 6, pp. 471-491, 2000.

[15] D. Manolis, C. Diakaki, I. Papamichail et al., "Simulation investigations of the coordinated traffic-responsive signal control strategy TUC with actuation at the local junction level," European Transport Research Review, vol. 10, no. 2, pp. 25-37, 2018.

[16] S. Baldi, I. Michailidis, V. Ntampasi, E. Kosmatopoulos, I. Papamichail, and M. Papageorgiou, "A simulation-based traffic signal control for congested urban traffic networks," Transportation Science, vol. 53, no. 1, pp. 6-20, 2019.

[17] Z.-S. Hou and J.-X. Xu, "On data-driven control theory: the state of the art and perspective," Acta Automatica Sinica, vol. 35, no. 6, pp. 650-667, 2009. 
[18] T. Oda, "Signal control by successive updating of control parameters based on prediction of traffic flow," Electrical Engineering in Japan, vol. 161, no. 3, pp. 49-57, 2007.

[19] B. Placzek, "A self-organizing system for urban traffic control based on predictive interval microscopic model," Engineering Applications of Artificial Intelligence, vol. 34, pp. 75-84, 2014.

[20] D. Heng, W. H. Zhang, X. Y. Zhang et al., "Multi-state flow signal control based on traffic prediction," China Journal of Highway and Transport, vol. 25, no. 5, pp. 126-133, 2012.

[21] J. M. Xu, X. L. Zou, and Y. Y. Ma, "Self-adaptive control of isolated intersection based on short-term traffic flow prediction," Journal of Chongqing Jiaotong University (Natural Science), vol. 38, no. 4, pp. 9-14, 2018.

[22] G. Abu-Lebdeh, "Integrated adaptive-signal dynamic-speed control of signalized arterials," Journal of Transportation Engineering, vol. 128, no. 5, pp. 447-451, 2002.

[23] Y. Bowen, "A traffic efficiency promotion algorithm for urban arterial roads based on speed guidance," in Proceedings of the International Conference on Connected Vehicles and Expo, pp. 869-873, Las Vegas, NV, USA, December 2013.

[24] P. Chen, C. Yan, J. Sun, Y. Wang, S. Chen, and K. Li, "Dynamic eco-driving speed guidance at signalized intersections: multivehicle driving simulator based experimental study," Journal of Advanced Transportation, vol. 2018, Article ID 6031764, 11 pages, 2018.

[25] H. Liu, L. Yang, S. Shao, and Z. Wang, "Speed guidance strategies at signalized intersections in a vehicle infrastructure cooperative environment," Journal of Chongqing Jiaotong University (Natural Science), vol. 38, no. 12, pp. 8-17, 2019.

[26] L. Zhang, Q. Zhao, L. Wang, and L. Zhang, "Urban intersection signal control based on time-space resource scheduling," IEEE Access, vol. 9, no. 9, pp. 49281-49291, 2021.

[27] G. Jiang, Y. Liang, and J. Guan, "Bus signal and VIP vehicle emergency priority at intersections in beijing olympic center," Journal of Transportation Systems Engineering and Information Technology, vol. 8, no. 6, pp. 101-106, 2008.

[28] Li Wang, L. Zhang, Ke Pan, and Z. Li, "Traffic signal switching control approach based on state control ability aanlysis," Journal of Zhejiang University (Engineering Science), vol. 50, no. 7, pp. 1266-1275, 2016. 